\title{
Clinicopathological analysis of colorectal carcinoid tumors and patient outcomes
}

\author{
Hung-Hsin Lin ${ }^{1,2}$, Jen-Kou Lin 1,2, Jeng-Kai Jiang 1,2, Chun-Chi Lin ${ }^{1,2}$, Yuan-Tzu Lan ${ }^{1,2}$, Shung-Haur Yang 1,2, \\ Huann-Sheng Wang ${ }^{1,2}$, Wei-Shone Chen ${ }^{1,2}$, Tzu-Chen Lin', Wen-Yih Liang ${ }^{2,3}$ and Shih-Ching Chang ${ }^{1,2^{*}}$
}

\begin{abstract}
Background: Colorectal carcinoid tumors are often described as being low-grade malignant. The objective of the current study was to address the clinicopathological features and outcomes of patients with colorectal carcinoid tumors.

Methods: A total of 63 patients with colorectal carcinoid tumors were identified and evaluated using surgical pathology files and medical records between January 2000 and June 2012 at the Veterans General Hospital, Taipei, Taiwan.

Results: The median age of the 63 patients was 57.0 years; 38 (60.3\%) were male and 25 (39.7\%) female. The rectum was the most common tumor site (90.5\%). Tumor size was $10.8 \pm 7.4 \mathrm{~mm}$, ranging from 2 to $50 \mathrm{~mm}$ in diameter. There were 40 patients (63.5\%) who received endoscopic treatment for a tumor size of $7.7 \pm 4.0 \mathrm{~mm}$, 15 (23.8\%) who underwent transanal excision for a mean size of $9.2 \pm 4.5 \mathrm{~mm}$ and eight (12.7\%) who underwent radical surgical resection (mean size: $29.5 \pm 13.0 \mathrm{~mm}$ ). Lymph node metastasis was significantly associated with tumor size. Totally distant metastases (liver) were demonstrated in four (6.3\%), patients with mean tumor size of $31.3 \pm 9.4 \mathrm{~mm}$ (20 to $50 \mathrm{~mm}$ ). The extent of the disease was associated with survival and the five-year overall survival rate was $92.1 \%$.

Conclusions: With widespread colorectal cancer screening, heightened awareness and improved diagnostic modalities, the incidence of colorectal carcinoid tumors will continue to increase. We demonstrated that small-sized colorectal carcinoid tumors and those localized in the mucosa or submucosa may be safely and effectively removed via endoscopic or transanal local excision.
\end{abstract}

Keywords: Colorectal, Carcinoid tumor, Lymph node metastasis, Clinicopathological

\section{Background}

Carcinoid tumors are rare, comprising approximately $0.49 \%$ of all malignancies [1]. These are the most common neuroendocrine tumors in the gastrointestinal system and most incidences occur in the gastrointestinal tract [2], with the rectum and ileum as the most prevalent tumor sites $[1,3]$. Gastrointestinal carcinoids are currently referred to as gastroenteropancreatic neuroendocrine tumors (GEP-NETs). Despite several re-classifications of GEP-

\footnotetext{
* Correspondence: changsc@vghtpe.gov.tw

'Division of Colon and Rectal Surgery, Department of Surgery, Taipei Veterans General Hospital, No 201, Sec. 2, Shih-Pai Road, Taipei 11217, Taiwan

${ }^{2}$ National Yang-Ming University, No.155, Sec.2, Linong Street, Taipei 11221 Taiwan

Full list of author information is available at the end of the article
}

NETs by the World Health Organization (WHO), the term 'carcinoid' is still used as a synonym for 'well-differentiated NET' and the term 'malignant carcinoid' is used as a synonym for 'well-differentiated neuroendocrine carcinoma'. Among such tumors, carcinoids of the colon and the rectum are grouped together in the WHO classification and are distinguished from those of the appendix or the ileum. In the WHO classification, colorectal carcinoids are described as 'low-grade malignant', even in the presence of metastasis [4]. Furthermore, the WHO classification defines colorectal carcinoids as 'benign' if the tumors are localized in the submucosa, measure $20 \mathrm{~mm}$ or less and lack vascular invasion [4]. Although most colorectal carcinoids are localized at the time of diagnosis and have low malignant potential, 
rectal carcinoids measuring less than $1 \mathrm{~cm}$ in size still have malignant potential, and the recorded incidence of metastasis for these tumors ranges from 1.7 to $3.4 \%[5,6]$.

Results related to crucial determinants for metastasis in colorectal carcinoids possibly vary in relation to the small number of cases available in each study. Numerous studies have reported various indicators for metastasis, including a tumor size of $10 \mathrm{~mm}$ or more or $20 \mathrm{~mm}$ or more, invasion to the muscularis propria, older age, male gender, tumor site, histological growth pattern and mitotic rate [7]. As a result, there has been a disagreement on the therapeutic strategy for use in colorectal carcinoids, particularly in relation to whether local excision or radical resection is suitable for an intermediate tumor size between 10 and $20 \mathrm{~mm}$ [8].

The purpose of this study is to review and analyze the clinicopathological features of carcinoid tumors of the colon and rectum at a single institution over a 12-year period.

\section{Methods}

\section{Patients and clinical data}

We retrospectively identified patients using our institution's pathology database and obtained the corresponding medical records. A total of 63 patients with colorectal carcinoids were enrolled at the Taipei Veterans General Hospital between January 2000 and June 2012. The investigation was approved by the local ethics committee.

Clinical data were prospectively recorded in detail and stored in electronic files. The data base included: (1) name, gender, age, family history and major medical problems of each patient; (2) location, size, gross appearance, stage, differentiation and important pathological prognostic features of the tumor and (3) type of operation, complications, recurrence and follow-up conditions. Pathologic staging of the disease was performed according to the American Joint Committee on Cancer (AJCC) Staging Manual, seventh edition, after review of the surgical specimen and investigation of distant metastases [4].

Rectal carcinoids were defined as tumors located within $15 \mathrm{~cm}$ of the anal verge, whereas tumors more than $15 \mathrm{~cm}$ above the anal verge were regarded as colonic carcinoids. The tumors were classified into three groups according to tumor size: (1) a tumor diameter of less than $10 \mathrm{~mm}$; (2) a tumor diameter between 10 and $20 \mathrm{~mm}$ and (3) a tumor diameter of over $20 \mathrm{~mm}$. Tumor sizes were confirmed through pathology reports. In cases where the tumor size was not clearly described in the pathology report, the size was determined according to the colonoscopy report. Treatment methods were classified as endoscopic resection, transanal resection and radical operation.

All patients were followed up on between three and six months after surgery during the first three years, and annually thereafter. Digital examination, chest X-ray, abdominopelvic ultrasound or computed tomography (CT) scan were used. Recurrence was defined as a local or distant disease diagnosed more than three months after the initial surgery, proven by pathological confirmation or progressively increasing size in imaging studies. Survival time was defined as the time elapsed from the date of diagnosis of the carcinoid tumor until death from all causes, or until 30 November 2013, which was the final date of the analysis used in this study.

\section{Statistical analysis}

The statistical endpoint used in our analysis was patient overall survival from the date of treatment. Group distribution for each clinicopathological trait was compared using the two-tailed Fisher's exact test and the chi-square test. Numerical values were compared using the Student's $t$-test and data are expressed as mean \pm standard deviation (SD). Kaplan-Meier survival curves were constructed and compared using the log-rank test and multivariate analysis was performed using the Cox proportional hazard model. Statistical analyses were performed using the SPSS package (version 16.0 for Windows, SPSS, Chicago, Illinois, United States).

\section{Results}

A total of 63 patients were enrolled in this study and the population consisted of 38 males $(60.3 \%)$ and 25 females (39.7\%). The mean age at tumor resection was $57.0 \pm$ 12.5 years (range: 26 to 87 years) and the male-to-female ratio was $1.52: 1$. The mean tumor diameter was $10.8 \pm$ $7.4 \mathrm{~mm}$ (range: 2 to $50 \mathrm{~mm}$ ). The clinicopathological features are outlined in Table 1 and include patient age at diagnosis, gender, tumor location, tumor size and treatment (endoscopic resection, local excision and radical operation). The distribution of surgical methods and tumor size of colorectal carcinoid tumors is showed in Table 2. No patient had carcinoid syndrome at diagnosis. At diagnosis, 49 patients (77.8\%) were asymptomatic. The most common presenting symptom was bloody stool (six patients, 9.8\%). The other main symptoms included diarrhea (three patients, 4.9\%), weight loss (two patients, $3.3 \%$ ), a palpable anal mass (two patients, $3.3 \%$ ) and constipation (one patient, 1.6\%).

A total of nine patients were diagnosed as having lymph node metastasis by pathologic investigation or imaging studies. Six patients who underwent low anterior resection (LAR) were identified by histological examination as having lymph node metastasis, and two patients who underwent only endoscopic resection because of unresectable distant metastasis were suspected of having lymph node metastasis on the basis of CT scans. One patient suffered obstruction of the colon and underwent a transverse loop colostomy, but later died from unresectable distant metastasis. 


\begin{tabular}{|c|c|c|c|c|}
\hline & $\begin{array}{l}\text { Total } \\
(n=63)\end{array}$ & $\begin{array}{l}\text { Rectum } \\
(n=57)\end{array}$ & $\begin{array}{l}\text { Colon } \\
(n=6)\end{array}$ & $P$ value \\
\hline Age (mean $\pm S D$, years) & $57.0 \pm 12.5$ & $57.2 \pm 12.6$ & $54.5 \pm 10.8$ & 1.000 \\
\hline$<65$ & $42(66.7)$ & $38(66.7)$ & $4(66.7)$ & \\
\hline$\geq 65$ & $21(33.3)$ & 19 (33.3) & $2(33.3)$ & \\
\hline Gender & 1.52: 1 & & & 0.738 \\
\hline Male & $38(60.3)$ & $34(59.6)$ & $4(66.7)$ & \\
\hline Female & $25(39.7)$ & $23(40.4)$ & $2(33.3)$ & \\
\hline $\begin{array}{l}\text { Tumor size } \\
(\text { mean } \pm S D, m m)\end{array}$ & $10.8 \pm 7.4$ & $10.1 \pm 6.4$ & $17.2 \pm 15.2$ & 0.414 \\
\hline$<10$ & $41(65.1)$ & $37(65.0)$ & $4(66.7)$ & \\
\hline $10-20$ & $10(15.9)$ & $10(17.5)$ & $0(0.0)$ & \\
\hline$\geq 20$ & $12(19.0)$ & $10(17.5)$ & $2(33.3)$ & \\
\hline Treatment & & & & 0.149 \\
\hline Endoscopic resection & $40(63.5)$ & $36(63.2)$ & $4(66.7)$ & \\
\hline Local excision & $15(23.8)$ & $15(26.3)$ & $0(0.0)$ & \\
\hline Radical operation & $8(12.7)$ & $6(10.5)$ & $2(33.3)$ & \\
\hline
\end{tabular}

The values in parentheses are presented as percentages.

Metastasis to the lymph nodes was significantly associated with tumor size. No lymph node metastasis was observed for tumors smaller than $10 \mathrm{~mm}, 10 \%$ lymph node metastasis was observed for tumors between 10 and $20 \mathrm{~mm}$ and $58.3 \%$ was observed for tumors larger than $20 \mathrm{~mm}$ (Table 3). Distant metastasis at diagnosis was assessed through CT scans or pathological examinations in four cases. All patients with distant metastasis had tumors larger than $20 \mathrm{~mm}$ (Table 4). Results of the TNM stage classification according to the AJCC are shown in Table 5.

The mean length of the follow-up period in this study was 77.1 months. Two patients were lost to follow-up. The five-year overall survival rate was $92.1 \%$. For patients with tumors smaller than $20 \mathrm{~mm}$, the five-year overall survival rate was $94.0 \%$, which was significantly better than those with tumors larger than $20 \mathrm{~mm}(83.3 \% ; P=0.003)$ (Figure 1). However, if the cut-off value of the tumor size was defined as $10 \mathrm{~mm}$, the five-year overall survival rates

Table 2 The distribution of surgical methods and tumor size of colorectal carcinoid tumors

\begin{tabular}{lccc}
\hline Tumor size $(\mathbf{m m})$ & \multicolumn{3}{c}{ Surgical methods } \\
\cline { 2 - 4 } & Endoscopic & Local excision & Radical operation \\
\hline$<10$ & 30 & 10 & 1 \\
$10-20$ & 7 & 2 & 1 \\
$>20$ & 3 & 3 & 6 \\
Total & 40 & 15 & 8 \\
\hline
\end{tabular}

Table 3 Rate of lymph node metastasis in 63 colorectal carcinoid tumors

\begin{tabular}{lccc}
\hline Tumor size $(\mathbf{m m})$ & Total $(\mathbf{n = 6 3})$ & Rectum $(\mathbf{n}=\mathbf{5 7})$ & Colon $(\mathbf{n}=\mathbf{6})$ \\
\hline$<10$ & $0 / 41$ & $0 / 37$ & $0 / 4$ \\
$10-20$ & $1 / 10(10.0)$ & $1 / 10(10.0)$ & - \\
$>20$ & $7 / 12(58.3)$ & $7 / 10(70.0)$ & $0 / 2$ \\
Total & $8 / 63(12.7)$ & $8 / 57(14.0)$ & $0 / 6$ \\
\hline \multicolumn{7}{l}{ The values in parentheses are presented as percentages. }
\end{tabular}

were observed to be similar between patients with tumors larger than, and less than $10 \mathrm{~mm}$ (90.9 versus $92.1 \%$; $P=0.08$ ).

For the 10 patients with tumors between 10 and $20 \mathrm{~mm}$, seven patients underwent colonoscopic resection, two patients underwent transanal excision, and one patient received radical low anterior resection. During the follow-up period, a 76-year-old male patient developed local recurrence in the rectum and underwent low anterior resection for a $2.0-\mathrm{cm}$-sized rectal carcinoid. The original pathology revealed invasion of the muscularis propria and two out of 12 lymph nodes were positive for metastasis.

Overall, seven patients in the cohort (11.1\%) had a synchronous second primary tumor at the time of carcinoid diagnosis. This included five patients with colorectal adenocarcinoma, one patient with pancreatic adenocarcinoma and one patient with endometrial adenocarcinoma.

\section{Discussion}

In general, the incidence of carcinoid tumors of the colon is reported to be very low and comprises between 4 and $8 \%$ of all carcinoid tumors in the digestive system $[1,8,9]$. With widespread colorectal cancer screening, heightened awareness and improved diagnostic modalities, the incidence of early detection of colorectal carcinoid tumors should continue to increase. Although our study was limited to the colon and the rectum, a tumor incidence of 9.5\% was observed in the colon, which is slightly higher than the generally accepted incidence rate. The predominance of males over females with rectal carcinoids was observed by Yoon et al. [10] (ratio 1:15), Konishi et al. [8] (ratio 1:2.2), and Jetmore et al. [11] (ratio 1:1.7).

Table 4 Rate of distant metastasis in 63 colorectal carcinoid tumors

\begin{tabular}{lccc}
\hline Tumor size $(\mathbf{m m})$ & Total $(\mathbf{n}=\mathbf{6 3})$ & Rectum $(\mathbf{n}=\mathbf{5 7})$ & Colon $(\mathbf{n}=\mathbf{6})$ \\
\hline$<10$ & $0 / 41$ & $0 / 37$ & $0 / 4$ \\
$10-20$ & $0 / 10$ & $0 / 10$ & - \\
$>20$ & $4 / 12(33.3)$ & $4 / 10(40.0)$ & $0 / 2$ \\
Total & $4 / 63(6.3)$ & $4 / 57(7.0)$ & $0 / 6$ \\
\hline
\end{tabular}

The values in parentheses are presented as percentages. 
Table 5 TNM staging of colorectal carcinoid tumors according to AJCC, seventh edition $(n=63)$

\begin{tabular}{lrc}
\hline & TNM stage (clinical or pathological) & Number (\%) \\
\hline & T1aNOM0 & $41(65.1)$ \\
Ila & T1bNOM0 & $9(14.3)$ \\
Ilb & T2NOMO & $4(6.3)$ \\
Illa & T3NOMO & $1(1.6)$ \\
IIIb & T4NOM0 & 0 \\
IV & Any T, N1M0 & $4(6.3)$ \\
\hline
\end{tabular}

$\mathrm{T} 1$, tumor invades lamina propria or submucosa and size $\leq 2 \mathrm{~cm} ; \mathrm{T} 1 \mathrm{a}$, tumor size $<1 \mathrm{~cm}$ in greatest dimension; $T 1 \mathrm{~b}$, tumor size 1 to $2 \mathrm{~cm}$ in greatest dimension; T2, tumor invades muscularis propria or size $>2 \mathrm{~cm}$ with invasion of lamina propria or submucosa; 13 , tumor invades through the muscularis propria into the subserosa, or into non-peritonealized pericolic or perirectal tissues; T4, tumor invades peritoneum or other organs; N0, no regional lymph node metastasis; $\mathrm{N} 1$, regional lymph node metastasis; $\mathrm{M0}$, no distant metastasis; M1, distant metastasis.

In addition, the same predominance was observed in our study (ratio 1:1.52). Carcinoid syndrome was not observed in the patients in our study, which corresponds to prior observations that most colorectal carcinoids are nonfunctioning and that carcinoid symptomatology is rare (less than 5\%) [7].

In the recently revised AJCC cancer staging, carcinoid tumors are classified as a malignant disease. The stage classifications are based on tumor size, the involved layer of the bowel wall and the presence or absence of lymph node or distant metastasis. According to this staging system, our series of cases are classified into 48 stage I cases, four stage IIa cases, one stage IIb case, no IIIa cases, four stage IIIb cases and four stage IV cases. The staging results reflect that most cases were early lesions and were incidentally detected by colonoscopy screening.

A population-based study in Japan reported lymph node metastasis of $3.7 \%$ for rectal carcinoids with sizes of $5 \mathrm{~mm}$ or less, and $9.7 \%$ lymph node metastasis for tumors with sizes of less than $10 \mathrm{~mm}$ [9]. These results complicate decisions regarding treatment methods, even in small-sized rectal carcinoids. In our study, only one patient with a rectal carcinoid size between 10 and $20 \mathrm{~mm}$ developed regional lymph node metastasis (10.0\%). However, if lymph node metastasis is to be accurately predicted, additional studies on the risk factors are required and treatment methods should be determined considering various risk factors $[2,12]$.

Although tumor size is often cited as the most important prognostic indicator for carcinoid tumors, the best indicators are actually the evidence of invasive growth and the presence of regional or distant metastasis. In our study, no cases of distant metastasis in patients with a tumor size of less than $20 \mathrm{~mm}$ were observed. The fiveyear survival rates for patients with tumor sizes greater than and less than $20 \mathrm{~mm}$, were $83.3 \%$ and $94.0 \%$, respectively $(P=0.003)$. Li et al. [13] reported that rectal and sigmoid carcinoids measuring more than $2 \mathrm{~cm}$ or those that invaded muscle layers (T2) or beyond, or those with distant metastasis had significantly more frequent metastases and a worse survival rate. Patients with these tumors should be treated aggressively and should not be treated using excisional biopsy.

In the case of rectal carcinoids, the risk of lymph node metastasis and distant metastasis varies depending

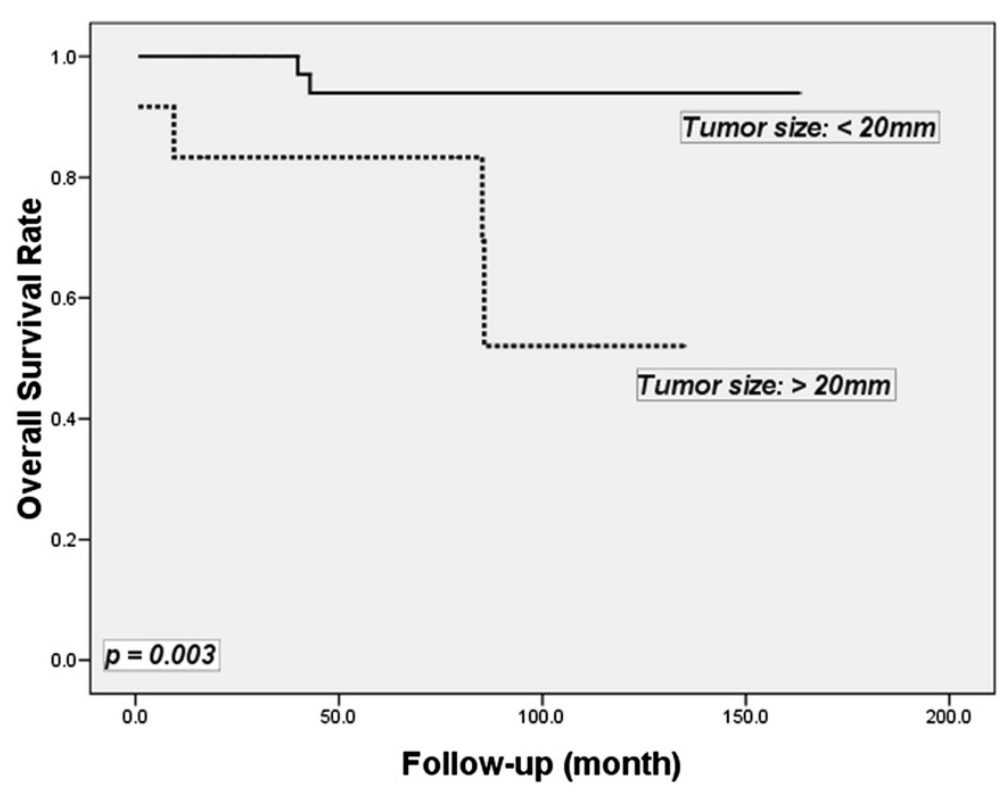

Figure 1 Kaplan-Meier plot illustrating five-year overall survival by tumor size. 
on tumor size. Thus, regarding tumor size is the most important factor in deciding an appropriate treatment method. The reported risk of lymph node metastasis for rectal carcinoids smaller than $10 \mathrm{~mm}$ is usually less than $3 \%$; thus, regional treatment including colonoscopic resection is appropriate [3]. However, radical surgery is recommended for tumors larger than $20 \mathrm{~mm}$, where the risk of metastasis is known to be 60 to $80 \%$. Shields et al. [14] reported that a tumor size of more than $10 \mathrm{~mm}$ and lymphovascular invasion were significantly associated with the presence of nodal disease, rendering mesorectal excision advisable. Treatment methods for tumors with sizes between 10 and $20 \mathrm{~mm}$ are controversial and no established treatment guideline exists.

In our present study, there was no lymph node metastasis observed in relation to tumors smaller than $10 \mathrm{~mm}$, $10 \%$ in relation to tumors between 10 and $20 \mathrm{~mm}$ and $58.3 \%$ in relation to tumors larger than $20 \mathrm{~mm}$. Although we are unable to draw specific treatment recommendations based on the results of the current study because of its retrospective nature and small numbers in each subgroup, it appears that rectal carcinoids measuring less than $10 \mathrm{~mm}$ can be safely managed by local excision. In addition, local excision is usually recommended for tumors between 10 and $20 \mathrm{~mm}$, but radical surgery should be considered if there is evidence of lymph node metastasis or lymphovascular invasion on biopsy. In contrast, for tumors larger than $20 \mathrm{~mm}$, radical surgery is mandatory. To rule out distant metastasis, imaging studies, such as CT or magnetic resonance imaging, are recommended for patients with rectal carcinoids larger than $20 \mathrm{~mm}$.

In this study, we discovered that seven patients (11.1\%) had a synchronous second primary tumor at the time of carcinoid diagnosis. Associated malignancies were frequently identified in conjunction with carcinoids in the rectum and/or sigmoid (18 of 141 patients; 12.8\%) [13]. Of all the gastrointestinal tract carcinoids, colorectal carcinoids are reported to have a rate of second primary malignancy in the range of 5 to $32 \%$. The most common site of associated non-carcinoid malignancies was the gastrointestinal tract, which involved between 32 and $62 \%$ of all tumors, followed by the genitourinary tract (range: 9 to 22\%) and the lung and/or bronchial system (range: 9 to 13\%) [11,13,15-18]. The etiology for high risk of second primary neoplasms associated with carcinoid tumors remains unclear. Thus, our data and those from other studies strongly suggest that when a carcinoid tumor is identified in a patient, there is a need for close surveillance of the gastrointestinal tract, respiratory system and genitourinary tract, and that long-term follow-up is recommended for patients in order to identify any delayed metastasis or secondary malignancies.

\section{Conclusions}

In this study, we demonstrated that patients with smallsized colorectal carcinoid tumors that are localized in the mucosa or submucosa may be safely and effectively removed via endoscopic or transanal local excision. Patients with colorectal carcinoid tumors generally have a good prognosis, but long-term follow-up is recommended for patients with carcinoid tumors in order to identify any delayed metastasis or secondary malignancies.

\section{Consent}

Written informed consent was obtained from the patient for publication of this report and any accompanying images. A copy of the written consent is available for review by the Editor-in-Chief of this journal.

\section{Abbreviations}

AJCC: American Joint Committee on Cancer; CT: Computed tomography; GEP-NETs: Gastroenteropancreatic neuroendocrine tumors; WHO: World Health Organization.

\section{Competing interests}

The authors declare that they have no competing interests.

\section{Authors' contributions}

All authors participated in the conception and design of the study. $\mathrm{HHL}$ performed the clinical analysis and drafted the manuscript. JKL, JKJ, CCL, YTL, SHY, HSW, WSC, and TCL participated in surgery and interpretation of data. WYL carried out the review of pathological records. SCC carried out revision and analysis and is the corresponding author. All authors read and approved the final manuscript.

\section{Author details}

${ }^{1}$ Division of Colon and Rectal Surgery, Department of Surgery, Taipei Veterans General Hospital, No 201, Sec. 2, Shih-Pai Road, Taipei 11217 Taiwan. ${ }^{2}$ National Yang-Ming University, No.155, Sec.2, Linong Street, Taipei 11221, Taiwan. ${ }^{3}$ Department of Pathology, Taipei Veterans General Hospital, No 201, Sec. 2, Shih-Pai Road, Taipei 11217, Taiwan.

Received: 20 August 2014 Accepted: 18 November 2014 Published: 30 November 2014

\section{References}

1. Modlin IM, Lye KD, Kidd M: A 5-decade analysis of 13,715 carcinoid tumors. Cancer 2003, 97:934-959.

2. Maggard MA, O'Connell JB, Ko CY: Updated population-based review of carcinoid tumors. Ann Surg 2004, 240:117-122.

3. Coloproctology CSGoKSo: Clinical characteristics of colorectal carcinoid tumors. J Korean Soc Coloproctol 2011, 27:17-20.

4. Edge SBBD, Compton CC: AJCC Cancer Staging Manual. 7th edition. New York: Springer; 2010

5. Fahy BN, Tang LH, Klimstra D, Wong WD, Guillem JG, Paty PB, Temple LK, Shia J, Weiser MR: Carcinoid of the rectum risk stratification (CaRRs): a strategy for preoperative outcome assessment. Ann Surg Oncol 2007, 14:1735-1743.

6. Koura AN, Giacco GG, Curley SA, Skibber JM, Feig BW, Ellis LM: Carcinoid tumors of the rectum: effect of size, histopathology, and surgical treatment on metastasis free survival. Cancer 1997, 79:1294-1298.

7. Modlin IM, Kidd M, Latich I, Zikusoka MN, Shapiro MD: Current status of gastrointestinal carcinoids. Gastroenterology 2005, 128:1717-1751.

8. Konishi T, Watanabe T, Kishimoto J, Kotake K, Muto T, Nagawa H: Prognosis and risk factors of metastasis in colorectal carcinoids: results of a nationwide registry over 15 years. Gut 2007, 56:863-868.

9. Soga J: Early-stage carcinoids of the gastrointestinal tract: an analysis of 1914 reported cases. Cancer 2005, 103:1587-1595.

10. Yoon SN, Yu CS, Shin US, Kim CW, Lim SB, Kim JC: Clinicopathological characteristics of rectal carcinoids. Int J Colorectal Dis 2010, 25:1087-1092. 
11. Jetmore AB, Ray JE, Gathright JB Jr, McMullen KM, Hicks TC, Timmcke AE: Rectal carcinoids: the most frequent carcinoid tumor. Dis Colon Rectum 1992, 35:717-725.

12. Kim BN, Sohn DK, Hong CW, Han KS, Chang HJ, Jung KH, Lim SB, Choi HS, Jeong SY, Park JG: Atypical endoscopic features can be associated with metastasis in rectal carcinoid tumors. Surg Endosc 2008, 22:1992-1996.

13. Li AF, Hsu CY, Li A, Tai LC, Liang WY, Li WY, Tsay SH, Chen JY: A 35-year retrospective study of carcinoid tumors in Taiwan: differences in distribution with a high probability of associated second primary malignancies. Cancer 2008, 112:274-283.

14. Shields CJ, Tiret E, Winter DC: Carcinoid tumors of the rectum: a multi-institutional international collaboration. Ann Surg 2010, 252:750-755.

15. Modlin IM, Sandor A: An analysis of 8305 cases of carcinoid tumors. Cancer 1997, 79:813-829.

16. Soga J: Carcinoids of the rectum: an evaluation of 1271 reported cases. Surg Today 1997, 27:112-119.

17. Tichansky DS, Cagir B, Borrazzo E, Topham A, Palazzo J, Weaver EJ, Lange A, Fry RD: Risk of second cancers in patients with colorectal carcinoids. Dis Colon Rectum 2002, 45:91-97.

18. Habal N, Sims C, Bilchik AJ: Gastrointestinal carcinoid tumors and second primary malignancies. J Surg Oncol 2000, 75:310-316.

doi:10.1186/1477-7819-12-366

Cite this article as: Lin et al: Clinicopathological analysis of colorectal carcinoid tumors and patient outcomes. World Journal of Surgical Oncology 2014 12:366.

\section{Submit your next manuscript to BioMed Central and take full advantage of:}

- Convenient online submission

- Thorough peer review

- No space constraints or color figure charges

- Immediate publication on acceptance

- Inclusion in PubMed, CAS, Scopus and Google Scholar

- Research which is freely available for redistribution 